\title{
Perovskite Photovoltachromic Supercapacitor With All-Transparent Electrodes
}

Feichi Zhou, $\perp^{\dagger, *}$ Zhiwei Ren, ${ }^{,}$, Yuda Zhao, ${ }^{\dagger, *}$ Xinpeng Shen, ${ }^{\dagger, *}$ Aiwu Wang," Yang Yang Li, "Charles Surya, ${ }^{\S}$ and Yang Chai ${ }^{*}$,

${ }^{\dagger}$ Department of Applied Physics, The Hong Kong Polytechnic University, Hung Hom, Kowloon, Hong Kong, People's Republic of China.

*Corresponding author: ychai@polyu.edu.hk

${ }^{\ddagger}$ The Hong Kong Polytechnic University Shenzhen Research Institute, Shenzhen, People's Republic of China

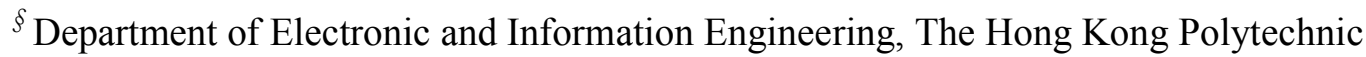
University, Hung Hom, Kowloon, Hong Kong, People's Republic of China

" Department of Physics and Materials Science, City University of Hong Kong

83 Tai Chee Rd., Kowloon, Hong Kong, People's Republic of China

$\perp$ These authors contributed equally to this work. 


\section{Supporting information:}

Figure S1 shows the fabrication process flow of a PSC. The absorption spectra and SEM image of the perovskite thin film are presented in Figure S2a and S2b. The 300-nm-thick perovskite thin film is well crystallized with good coverage and exhibits excellent light absorption over the wavelength ranging from $400 \mathrm{~nm}$ to $800 \mathrm{~nm}$. Figure S2c shows the $J-V$ curve of a typical PSC device with Au top electrode, which exhibits a high efficiency of $16.4 \%$.

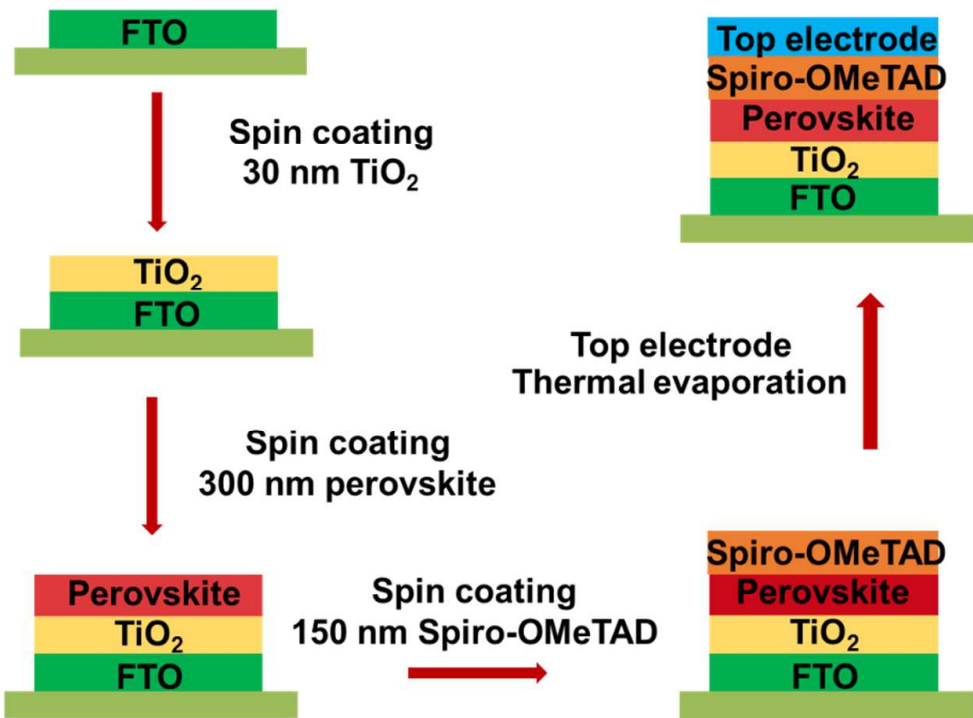

Figure S1. Fabrication process flow of the PSC. 
(a)

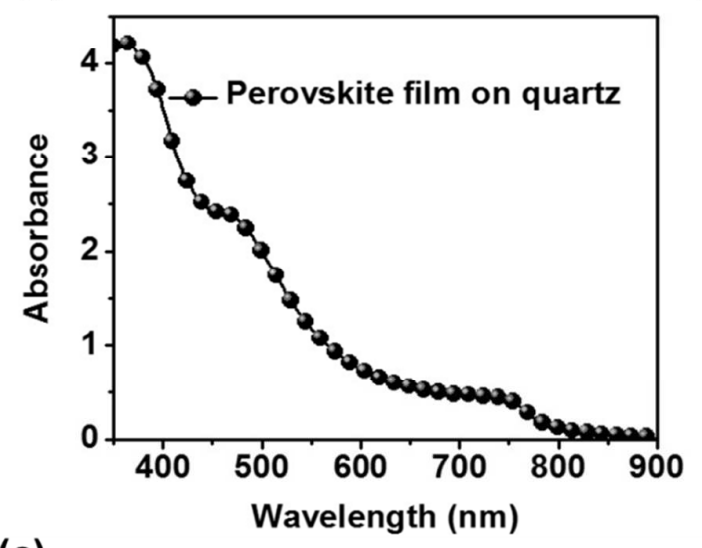

(c)

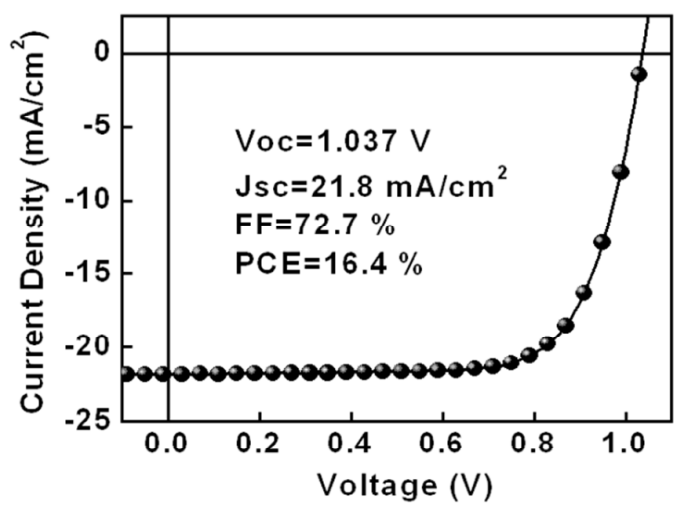

(b)

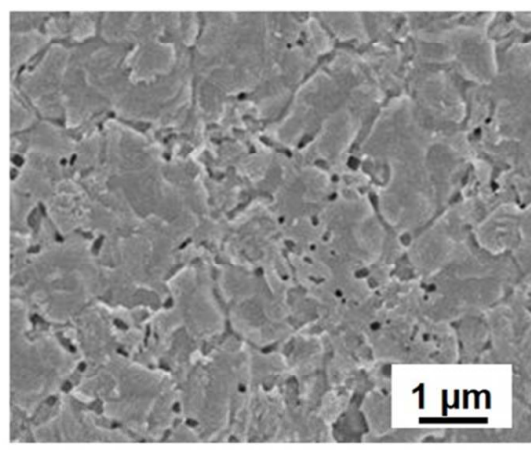

Figure S2. (a) Absorption spectra of perovskite film on quartz. (b) SEM image of perovskite film. (c) A J-V curve of a typical PSC device with Au top electrode, showing a PCE of $16.4 \%$.

Figure $\mathrm{S} 3 \mathrm{a}$ shows the schematic diagram of a symmetric $\mathrm{WO}_{3}$ electrochromic supercapacitor (ECS). A 200-nm-thick $\mathrm{WO}_{3}$ thin film was deposited onto an fluorine-doped tin oxide (FTO) substrate as supercapacitor electrode. Polyvinyl alcohol (PVA) doped with $1 \mathrm{M} \mathrm{H}_{2} \mathrm{SO}_{4}$ was used as the electrolyte. The symmetric ECS was assembled by two identical $\mathrm{WO}_{3}$ electrodes coated with PVA. Figure S3b and $\mathrm{S} 3 \mathrm{c}$ are representative atomic force microscopy (AFM) image and scanning 
electron microscopy (SEM) image of the as-deposited $\mathrm{WO}_{3}$ thin film, respectively. The nearly spherical grains are observed with a grain size of 50-200 $\mathrm{nm}$ and a roughness of $22.9 \mathrm{~nm}$. Lots of voids are presented between the grains, providing large surface area and effective paths for ion storage and redox reaction.Figure S3d and S3e show the X-ray photoelectron microscopy (XPS) spectra of the $\mathrm{WO}_{3}$ thin film. Four peaks are separated from the $\mathrm{W} 4 \mathrm{f}$ peak, corresponding to $\mathrm{W}^{6+}$ and $\mathrm{W}^{5+}$ oxidation states, respectively. The strong $\mathrm{W}^{6+}$ peaks suggest $\mathrm{W}^{6+}$ oxidation sate is the dominating state in the as-prepared $\mathrm{WO}_{3}$ thin film, which is in agreement with the transparent state. 
(a)

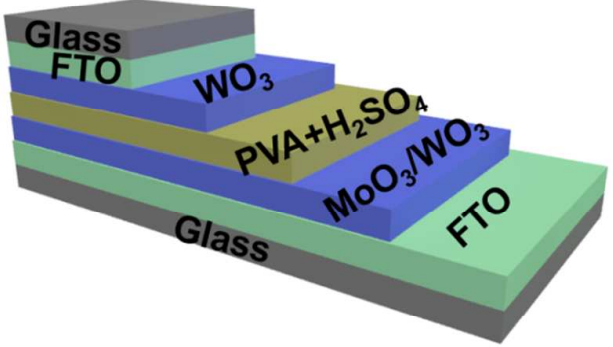

(c)

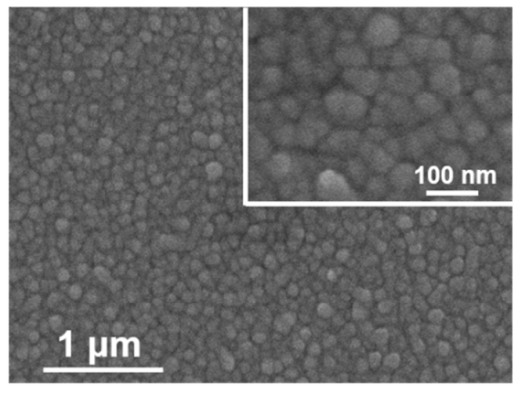

(e)

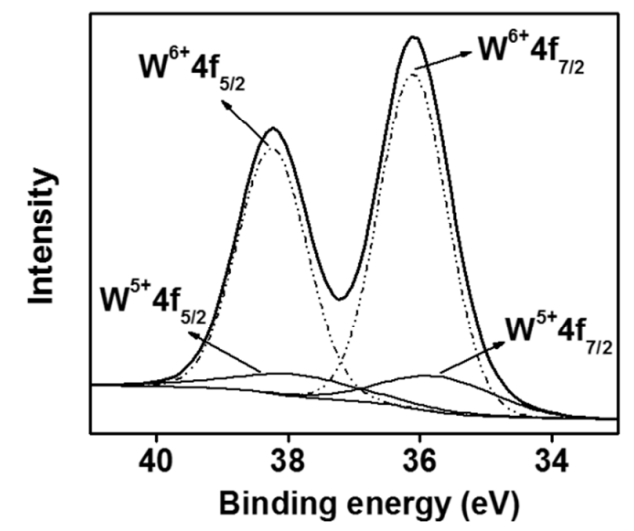

(b)

(d)
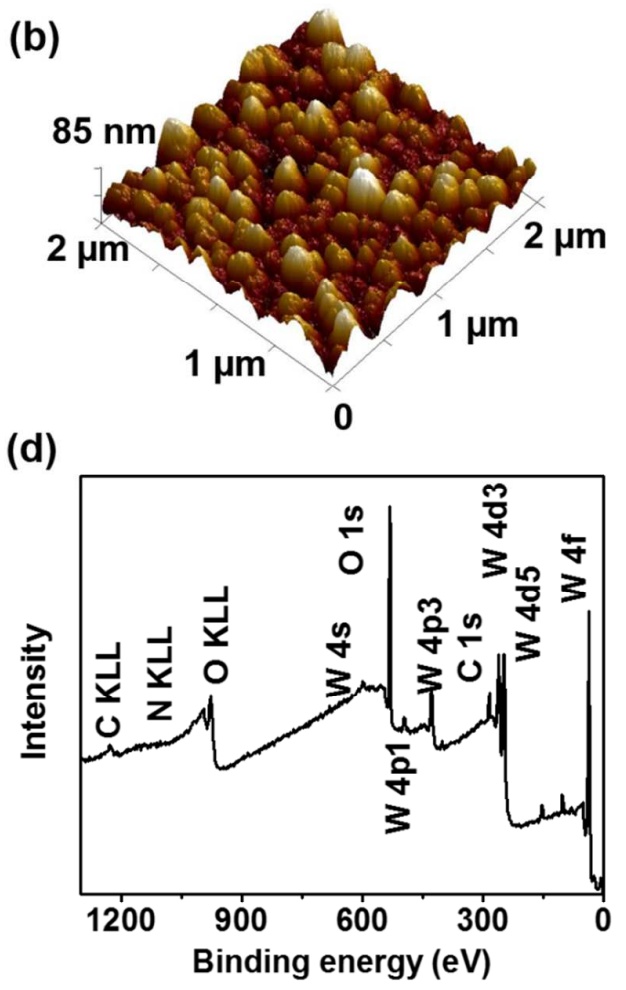

Figure S3. (a) Schematic diagram of a symmetric $\mathrm{WO}_{3}$ ECS. (b) AFM and (c) SEM image of the as-deposited $\mathrm{WO}_{3}$ thin film. (d) and (e) XPS spectra of the $\mathrm{WO}_{3}$ thin film.

Figure S4 (a), (b) and (c) shows the three-dimensional schematic, cross-section schematic and photo of co-anode PVCS. We use PDMS and epoxy to seal the PSC 
part in order to not damage the films in PSC. Figure S4 (d) and (e) show the three-dimensional schematic and photo of co-cathode PVCS.

(a)

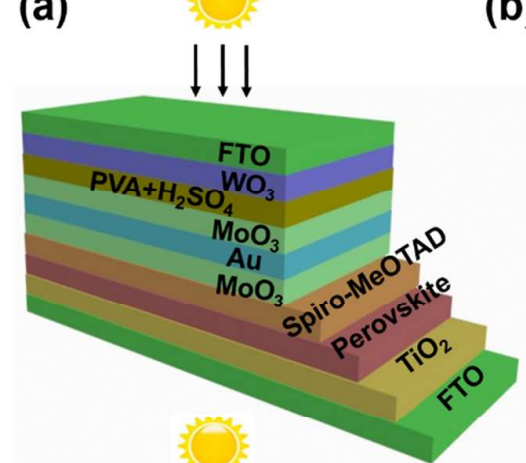

(d)
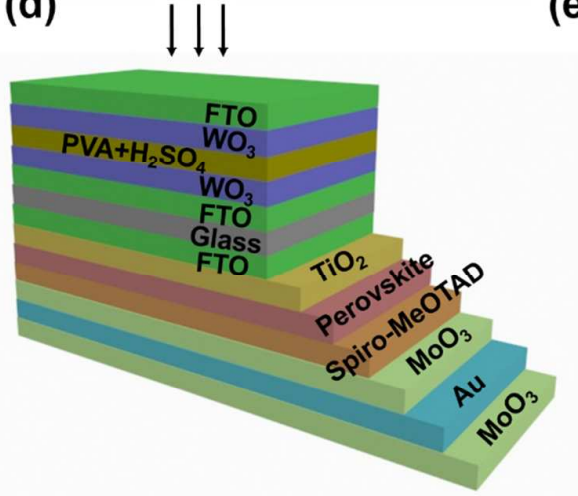

(b)

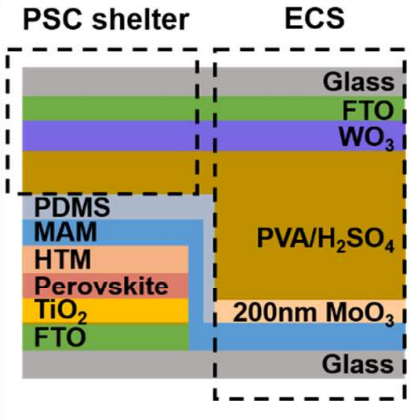

(e) (c)

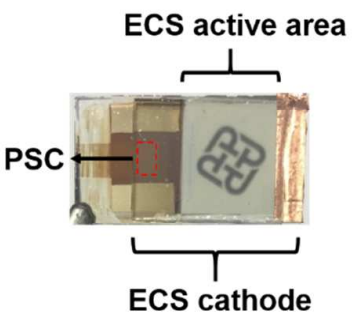

Figure S4. (a), (b) and (c) three-dimensional schematic, cross-section and photo of co-anode

PVCS. (d) and (e) three-dimensional schematic and photo of co-cathode PVCS. The red dashed boxes represent the active area of PSCs.

Figure S5 shows the the integration of the PSC with MAM top electrode and symmetric $\mathrm{WO}_{3}$ ECS connected by external wires. Figure S6a is the $J-V$ characteristic of a commercial $\mathrm{Si}$ solar cell. The commercial $\mathrm{Si}$ solar cell shows a $J_{s c}$ of 17.18 $\mathrm{mA} / \mathrm{cm}^{2}$ and a $V_{o c}$ of $1.34 \mathrm{~V}$. The ECS was charged by Si solar cell through external wire connection. The ECS was charged to $0.8 \mathrm{~V}$ within $8.5 \mathrm{~s}$ and reached its 
saturation within $35.0 \mathrm{~s}$, as shown in the Figure S6b. The energy storage response is faster than the ECS charge by PSC. The energy density, power density and total photoelectric conversion efficiency of this integrated device is calculated as 61.2 $\mathrm{mWh} / \mathrm{m}^{2}, 432.0 \mathrm{~mW} / \mathrm{m}^{2}$ and $5.51 \%$. These values for the device integrating PSC and ESC by external wire connection are $35.9 \mathrm{mWh} / \mathrm{m}^{2}, 461.5 \mathrm{~mW} / \mathrm{m}^{2}$ and $5.35 \%$, respectively, which are comparable with the charging by the commercial solar cell. In the meantime, the PSC provides widely tunable optical transmittance. The higher energy density of ECS charged by Si solar cell can be attributed to a higher charging voltage provided by the commercial Si solar cell. Thus, the ECS and PSC integrated device is promising to be a substitute of Si based integrated energy storage device for the lower cost in the PSC fabrication.

(a)

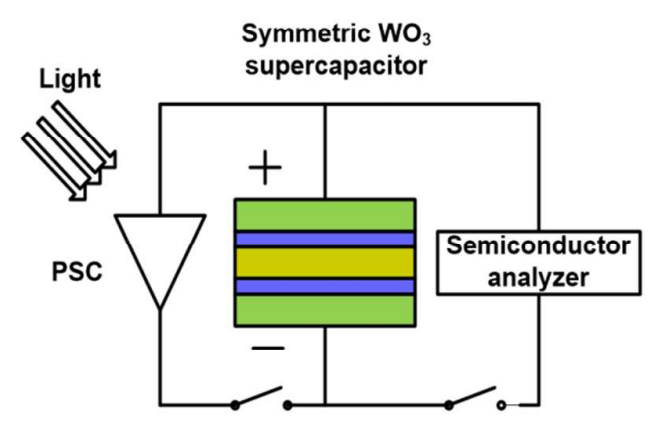

(b)

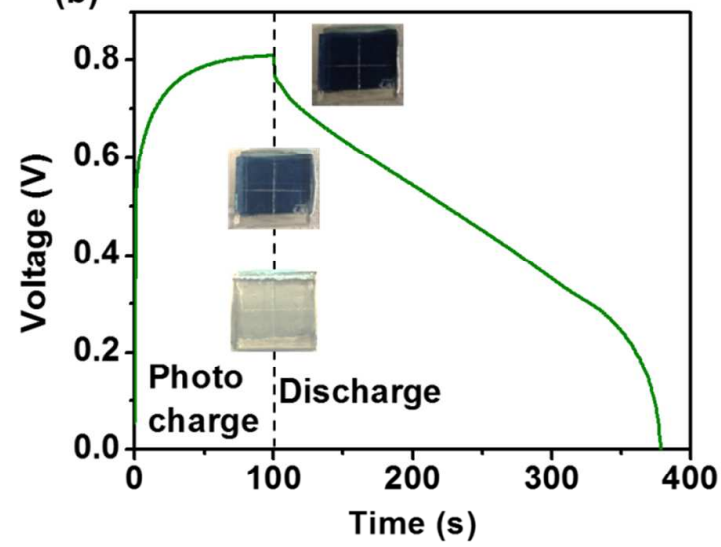

Figure S5. (a) Schematic illustration of the integration of the PSC with MAM anode and symmetric $\mathrm{WO}_{3}$ ECS connected by external wires. (b) V-t curves of photo-charging and discharging processes and the color changes during the processes. 

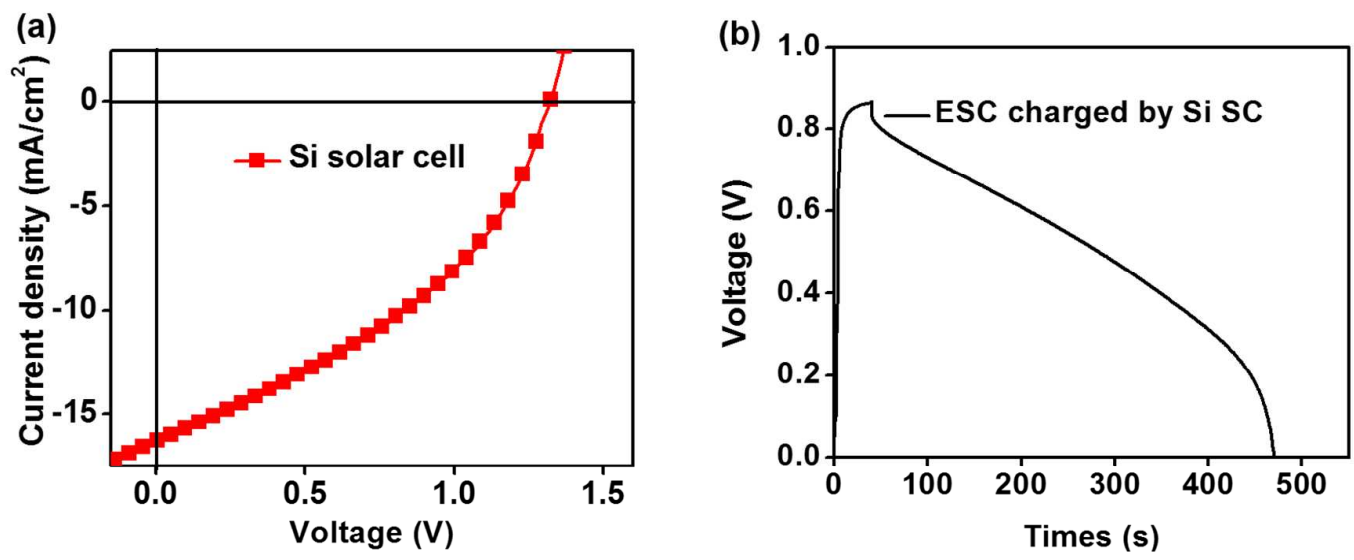

Figure S6. (a) A J-V curve of a commercial Si solar cell. (b) Photo-charging and discharging V-t curves of symmetric $\mathrm{WO}_{3}$ ECS integrated with the commercial Si solar cell.

Figure S7a shows the areal capacitances at different current densities. The areal capacitance of the device reaches $119.5 \mathrm{~F} / \mathrm{cm}^{2}$ at a current density of $0.12 \mathrm{~mA} / \mathrm{cm}^{2}$. Figure $\mathrm{S} 7 \mathrm{~b}$ is the Ragone plot of the $\mathrm{WO}_{3} \mathrm{ECS}$, which demonstrates a average power density of $6.8 \mathrm{~W} / \mathrm{m}^{2}$ at a charging current density of $1.20 \mathrm{~mA} / \mathrm{cm}^{2}$, and a energy density of $14.8 \mathrm{Wh} / \mathrm{m}^{2}$ at a charging current density of $0.12 \mathrm{~mA} / \mathrm{cm}^{2}$. 
(a)

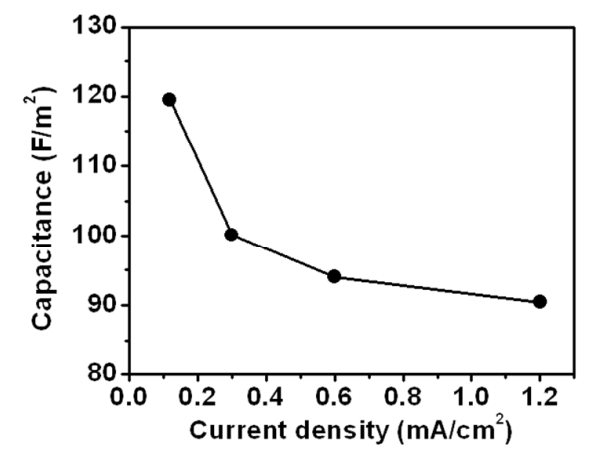

(b)

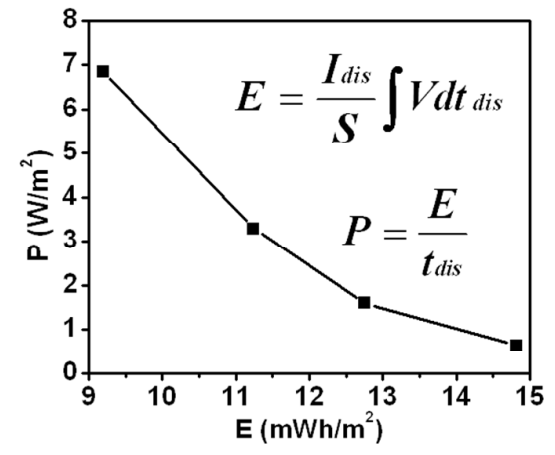

Figure S7. (a) The areal capacitances as a function of different current densities. (d) The Ragone plot of $\mathrm{WO}_{3}$ ECS, showing the relationship between power density and energy density.

We calculated the specific capacitance and energy density according to the discharging processes. According to the discharge curves, the total energy density of device can be calculated as:

$$
E_{\text {total }}=\int U d Q=\int U I d t_{d i s}=I \int U d t_{d i s}=I S(1)
$$

Where $\mathrm{I}$ is the discharge current density, $\mathrm{U}$ is the charged voltage, $t_{\text {dis }}$ is the total discharge time and $\mathrm{S}$ is the enclosed area of the discharge curve and coordinate axis.

The average power density can be expressed as:

$$
P_{t}=\frac{E_{\text {total }}}{t_{\text {dis }}}(2)
$$

Based on the calculated energy density, the supercapacitor areal capacitance can be calculated as:

$$
\begin{gathered}
E_{\text {total }}=\frac{1}{2} C U^{2}(3) \\
C=2 \frac{E_{\text {total }}}{U^{2}}(4)
\end{gathered}
$$

